\title{
Oral Manifestations of Vitamin B12 (Cobalamin) Deficiency: A Review
}

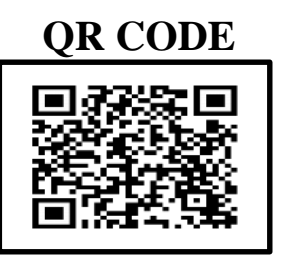

\section{KANAPARTHI ALEKHYA ${ }^{*_{1}}$, KATNE TEJASWI², SRIKAR MUPPIRALA³, DUKKIREDDY DIVYA4, L. KRISHNAPPA SOUJANYA5, RASHMITHA ARUTLA ${ }^{6}$}

Vitamin B12, also known as cobalamin is a water soluble vitamin. It is critical for normal functioning of the red blood cell formation and nervous system. It is a complex vitamin and it's deficiency is known to be one of the commonest deficiency in Indian population particularly in elderly and in vegans as it requires castles intrinsic factor released by parietal cells in the stomach for its absorption. Vitamin B12 deficiency may also result from pernicious anemia, gastrectomy \& intestinal malabsorption. It is often overlooked and may cause several oral, haematological, gastrointestinal, psychiatric and neurological manifestations. As it affects the oral mucosal tissues, early diagnosis of the manifestations may aid in diagnosing the underlying cause even before haematological examination. Hence, oral physicians should be vigilant enough to identify signs or symptoms of suspected vitamin deficiency in populations at risk. Early diagnosis is crucial for starting replacement therapy to avoid irreversible neurological damage. This article reviews the various oral manifestations of vitamin B12 deficiency and its management.

KEYWORDS: Vitamin B12 Deficiency, Megaloblastic Anemia, Cobalamine, Pernicious Anemia, Glossitis, Apthous Ulcer.

\section{INTRODUCTION}

A well balanced diet is the key to ensuring that the individuals receive optimal nutrients., ${ }^{1,2}$ Nutritional status and dietary habits can affect and be affected by all oral conditions. Nutrition interventions may be throughout of in terms of primary prevention, secondary prevention and tertiary precaution. ${ }^{3,4}$ Oral health cannot be exclusively maintained through one approach. The nutritional implications in dental condition are many and complex. Diet in India ranges from bland to spicy. Most Indian diet uses spices and variety of vegetables than global cuisines. If there is a deficit in these nutrients within the diet, malnutrition develops. It not only has an impact in the general health status of an individual but also affects the oral health.

Vitamins are a group of essential nutrients required in very minute amounts to participate and regulate chemical reactions within the body. Vitamins are not energy producing nutrients but, they enable energy to be released from carbohydrate, fat and protein for proper body functioning. IThe current life style changes and dietary habits also have an impact on the nutritional status of an individual. Nutritional deficiency, particularly due to vitamins can be identified at an early stage since, it affects the integrity of the oral cavity leading to progression of oral diseases. Vitamins further are classified into fat soluble and water soluble vitamins.
However, detailed review about all the vitamins is out of the scope of this article hence, this article focuses mainly on vitamin $\mathrm{B} 12$ as it is the most commonest deficiency seen in Indian population, particularly elderly \& population that follow the vegan diet.

A wide range of signs and symptoms may appear within the oral cavity of patients with B12 deficiencies due to changes in the underlining metabolism of epithelial cells. These changes may cause structural abnormalities in the cell altering the degree of keratinisation leading to a "beefy" tongue, glossitis, angular chelitis \& recurrent apthous ulcerations. ${ }^{1,3}$ It has been reported in literature that even vitamin B12 and folate deficiency have been found in patients with oral pre malignant lesions \&cancer.

\section{MATERIALS AND METHODS}

An extensive literature search was performed to create a comprehensive narrative review in the various oral manifestations of vitamin B12 deficiency and it's management. This was done by searching PubMed, Google Scholar, and Ovid for the key terms: "vitamin B12 deficiency" and "Cobalamin" or "oral manifestations of B12 deficiency." Randomized controlled studies were given first priority, followed by observational studies \& case reports. Systematic reviews, case studies and Cochrane reviews were included, and other non-English review sources were 
omitted. In the absence of controlled data specifically examining the oral manifestations due to vitamin B12 deficiencies, were extrapolated from the case reports. All relevant studies with high levels of supportive evidence were summarized and clinical symptoms under each category were elucted. It should be noted that most of the oral manifestations were reported as case reports \& case studies rather than controlled studies. Oral manifestations can be an early indicator of vitamin B12 deficiency even before haematological confirmation.

\section{VITAMIN B12 (COBALAMIN)}

Vitamin B12 is classified under water soluble vitamins and is considered to be unique among others due to its complex structure \& metabolism. Rickets isolated this vitamin in 1948. It is also called as anti-pernicious anemia vitamin or castle's extrinsic factor or erythrocyte maturation factor. It has been named as Cyanocobalamin as it is found as a coordination complex with cobalt ion and cyanide ion. The three main causes of low B12 levels are a lack of sufficient $\mathrm{B} 12$ in the diet, mal-absorption, and pernicious anemia. ${ }^{1,2,4}$

a) Normal absorption of vitamin B12: An understanding of the metabolic pathway of vitamin B12 absorption helps in identifying the potential causes of the deficiency. The acidic $\mathrm{pH}$ of the stomach initiates the breakdown of vitamin $\mathrm{B}_{12}$ bound to food. Intrinsic factor, released by parietal cells in the stomach binds to vitamin B12 in the duodenum. This complex of vitamin B12 intrinsic factor subsequently aids in the absorption of vitamin $\mathrm{B} 12$ in the terminal ileum. Hence, a state of vitamin B12 deficiency can also be due to malabsorption. ${ }^{4,5}$

b) Recommended dietary allowance: As recommended by NFI (2010) ${ }^{3}$,the dietary allowances per day are shown in the table below:

\begin{tabular}{|c|c|}
\hline Normal adults & $1.0 \mathrm{mcg}$ \\
\hline Pregnancy & $1.5 \mathrm{mcg}$ \\
\hline Lactation & $1.5 \mathrm{mcg}$ \\
\hline $\begin{array}{c}\text { Infants and } \\
\text { children }\end{array}$ & $0.2 \mathrm{mcg}$ \\
\hline
\end{tabular}

c) Sources: It is a unique vitamin as it is not found in any plant. It occurs primarily in animal foods, liver is the richest source. B12 is synthesized by microorganisms that grow in soil, water or the intestinal lumen of animals. The rich sources are meat, milk, curd \& eggs. Curd has considerably high amount of Lactobacillus that aids in synthesis of cyanocobalamine, hence it is a better source than milk. ${ }^{3}$

\section{d) Functions1of vitamin B12:}

1. Vitamin B12 plays an important role as in the synthesis of DNA within the bone marrow.

2. Vitamin B12 required for the enzymes that accomplish the synthesis and transfer of single carbon units like methyl group in enzymatic reaction. 3. For formation of mature RBC's.

\section{e) Deficiency:}

Dietary deficiency may arise in population who are strict vegetarians \& in patients who underwent gastrectomy . The symptoms include anorexia, weight loss, dyspnoea, prolonged bleeding time \& neurologic disturbances etc.

\section{GENERAL MANIFESTATIONS}

A deficiency due to dietary lack is uncommon on account of its wide spread occurrence in all animal foods and synthesis by intestinal flora. Of the various symptoms the most important deficiency symptom is macrocytic anaemia or pernicious anemia. It is also called as Addison's anemia or Biermer's anemia. It is characterized by low Hb levels, decreased number of erythrocytes and neurological manifestations. Degeneration of myelin sheath and peripheral nerves. It also has an impact on DNA replication leading to Ineffective heamotopoiesis. ${ }^{1,2}$

\section{ORAL MANIFESTATIONS}

As mentioned earlier vitamin B12 deficiency affects the oral health, it's oral manifestations reported in literature ${ }^{4}$ can be briefed as follows:

1. Beefy red tongue, which appears smooth \& glossy with glossopyrosis, glossitis and glossodynia.

2. Huntersglossitis or moellersglossitis similar to "Bald tongue of sandwith" seen in pellagra.

3. Aphthous ulceration,

4. Haemorrhagic gingiva, ulcerative gingivitis,

5. Epithelial dysplasia of oral mucosa, oral paresthesia \& Delayed wound healing. 
a. Vitamin B12 deficiency \& Glossitis: Vitamin B12 deficiency results in atrophic glossitis, which shows bright red, smooth, sore and burning tongue. ${ }^{1,4-8} \mathrm{Kim}$ $\mathrm{J}$ conducted a study in twenty two patients to compare clinical features of vitamin Bizdeficiency patients with a medical history of gastrectomy \& to those without a history of gastrectomy. Most reported manifestation was depapillation of the tongue. Oral symptoms responded to vitamin B12\& antifungal medications. They concluded that Vitamin B12deficiency should be considered in patients with glossodynia, in even those patients with normal oral mucosa with /without a history of gastrectomy. ${ }^{5}$

Stoopler ET et al., 2013 reported a case of 61-year-old woman with a 6-month history of a persistent burning sensation of the tongue. On clinical examination there was depapillation of the tongue and was diagnosed as glossitis. Laboratory investigations showed macrocytic anemia and low levels of vitamin $\mathrm{B} 12$ and the patient was advised injection $(1000 \mu \mathrm{g})$ of vitamin B12, which resulted in complete resolution of her symptoms. They suggested that glossits can be the only manifestation and the oral physician should treat the case accordingly. ${ }^{6}$

Pontes HA et al in 2009, reported a cases of a 41 year old female patient who was a strict vegetarian by diet. On clinical examination the oral findings revealed pale mucosa, atrophic glossitis and multiple erythematous areas on the dorsal and lateral borders of the tongue. On haematological examination there was severe B12 deficiency. The authors suggested that a through history and clinical examination aided in determining megaloblasticanemia. ${ }^{7}$

Graells J et al. in 2009, reported 4 cases of oral linear lesions associated with vitamin B12 deficiency free of neurologic symptoms and anaemia. They suggested that glossitis with linear lesions could be an early clinical manifestation and the serum levels have to be evaluated even in the absence of anaemia. ${ }^{8}$

\section{b. Vitamin B12 deficiency and recurrent aphthous stomatitis (RAS): Vitamin B12 acts as a co-enzyme for carbohydrate metabolism, protein synthesis and hematopoiesis. Recently, it has been suggested that changes within the oral mucosa like stomatitis \& glossitis may be the only early oral manifestation of vitamin B12deficiency.The exact role of vitamin B12 deficiency in the pathogenesis of recurrent aphthous stomatitis (RAS) is unclear.}

It has been hypothesised that in patients with RAS cell-mediated immunity is suppressed, and there are changes in the oral epithelium of the tongue and the buccal mucosa. These changes are similar to those seen in the bone marrow \& blood due to abnormal DNA synthesis. ${ }^{9-14}$

A study in 2013, was conducted by Liu HL et al. to determine the effectiveness of daily dosage in the management of adults with RAS. The results showed significant improvement of RAS in the study group compared with the control group suggesting that supplemental therapies may be beneficial in both short and long term management of RAS. ${ }^{9}$

Qazi JA et al. in 2011 conducted a study on 65 patients with RAS to confirm the beneficial effects of Vitamin B 12 in patients with RAS. Vitamin B12 500 mcg and 1000 mcg in sublingual dosage was administered for 6 months. The number, duration of pain, episodes of outbreak and the size of ulcer were reduced in group treated with Vitamin B12 100omcg irrespective of serum Vitamin B12 level compared to the other group with $500 \mathrm{mcg}$. The authors suggested that Vitamin B12 1000 mcg when administered sublingually is a safe $\&$ an effective treatment irrespective of their serum Vitamin B12 levels. ${ }^{10}$

Kozlak ST conducted a study in 2010 studied the effect of dietary vitamin intake in RAS patients. The results showed that the study group had lower daily intake of vitamin $\mathrm{B}_{12}$ as compared to the control group. The authors concluded that the presence of a deficiency allows the expression of an underlying tendency to ulceration \& dietary intake may reduce the episodes of outbreak of ulceration in RAS patients. ${ }^{11}$

Volkov I et al., 2009 conducted a study to identify the effectiveness of presents vitamin B12 in RAS. The authors concluded that a change in the local regulation of the cell-mediated immune system after activation and accumulation of cytotoxic T-cells might contribute to the localized breakdown of mucosa and also replacement therapy with vitamin B12 could be considerable benefit to patient. ${ }^{12}$

A randomized, double-blind, placebo-controlled trial was conducted in 2009, on 85 patients with RAS. The results suggested that the duration of outbreaks, number of ulcers, and pain were reduced at 5 and 6 months of treatment with vitamin B12. They 
concluded that supplemental treatment with vitamin B12 is a safe \& an effective method in patients suffering from RAS. ${ }^{13}$

A case-control study in 2006 was conducted on 143 patients with RAS. The results of the study showed that out of $37.8 \%$ patients with hematinic deficiencies, $26.6 \%$ showed less serum vitamin $\mathrm{B} 12$ in the control group \& $12.6 \%$ patients had low vitamin B12 level in study group. The authors concluded that hematinic deficiencies, particularly vitamin $\mathrm{B}_{12}$ deficiency are common in patients with recurrent aphthous stomatitis. They suggested that correction of these hematinic deficiencies could aid in the management of patients with RAS. ${ }^{14}$

c. Vitamin B12 deficiency and potentially malignant disorders (PMD'S) \& malignancy: Some aspects of diet are considered to be beneficial in the prevention of precancerous lesions and cancer. intake of food products rich in beta-carotene, vitamin $\mathrm{B}$, vitamin $\mathrm{E}$, vitamin $\mathrm{A}$, or their analogues have been reported to cause regression of pre-cancerous lesions like leukoplakia, further preventing progression to malignancy. ${ }^{15,16}$ Various studies have reported an association between low systemic levels of vitamin B12 and/or folate and an increased risk of malignancy in oral epithelial tissues in risk group. ${ }^{17-19}$

Previous studies suggested that cell kinetics \& cellular metabolism are associated with increased oxidative stress. ${ }^{17}$ Atrophic epithelium is the most commonly reported feature of various conditions and is considered to increase the risk of precancerous lesions and oral carcinoma. ${ }^{15-17,18}$ Among these micronutrients, vitamin B12 and folate are reported to have role in chemoprevention. ${ }^{18,19}$ It is also documented that supplementation of these micronutrients can prevent occurrence of neoplastic changes. ${ }^{18-22}$

Decreased plasma vitamin B12 and folate levels have been reported even due to certain exogenous compounds like tobacco smoke. ${ }^{23,24}$ Tobacco, is a known carcinogenic agent that has shown significant correlation with the decreased plasma vitamin B12 levels. ${ }^{18}$

d. Leukoplakia: Serum vitamin status was evaluated in 50 subjects with oral leukoplakia by Ramaswamy G et al. in 1996 and concluded that, except for vitamin $\mathrm{E}$, all the other vitamin levels were decreased in oral leukoplakias compared to the control groups. ${ }^{18}$ e. Head and Neck Squamous Cell Carcinoma (HNSC): Gorgulu O et al. in 2010 conducted a study on 60 subjects, to investigate the role of serum homocysteine, folate and vitamin B12 levels in the pathogenesis of laryngeal squamous cell cancer (LSCC) by measuring serum levels and suggested that these levels have an association in metabolic alterations in cellular metabolism leading to carcinogenesis. ${ }^{25}$

Almadori G et al. in 2005 studied the serum levels of vitamin B12, folate and homocysteine levels in patients with head and neck squamous cell carcinoma (HNSCC) and laryngeal leukoplakia. Their results suggested no statistically significant differences in serum vitamin B12 levels between study and control groups. However, they suggested that serum folate levels were significantly lower in patients with HNSCC and in patients with laryngeal leukoplakia. ${ }^{26}$

Raval GN et al. in 2002 evaluated vitamin B12 and folate status in 214 Head and Neck cancer and 167 subjects with oral precancerous conditions and suggested that the individuals with low levels of vitamin $\mathrm{B} 12$ and folate were at a higher risk of developing carcinoma \& also they reported significant correlation between vitamin B12 and folate levels in the subjects consuming tobacco. ${ }^{27}$

f. Oral submucous fibrosis (OSMF): Wang YP et al evaluated gastric parietal cell antibody positivity, iron, vitamin $\mathrm{B} 12$ and folic acid deficiencies in 68 OSMF patients. They suggested that 9 subjects had serum positivity, 6 subjects had vitamin B12 deficiency, 5 had folic acid deficiency and 2 with iron deficiency and concluded that there are high frequencies of vitamin $\mathrm{B} 12$ and folic acid deficiencies in OSMF patients. However the underlying reason for reduced serum levels in OSMF patients was unclear. ${ }^{28}$

g. Oral Lichen Planus (OLP): Association of deficiencies of iron, folic acid, and vitamin $\mathrm{B} 12$ and homocysteine level was assessed in 352 OLP subjects in a study conducted by Chen HM et al in 2015 and suggested that OLP patients had a higher frequency of Haemoglobin , iron or vitamin B12 deficiency and also abnormally elevated blood homocysteine level than control participants. ${ }^{29}$

Sahebjame $\mathrm{M}$ et al. in 2010 conducted a study to investigate the levels of serum Vitamin B12 and folic 
acids in patients with 48 OLP. The results suggested Vitamin B12 deficiency was found in 8 of 32 cases with OLP (25\%) while in the control group it was found in $12.5 \%$ of the subjects ( $\mathrm{p}>0.05)$. They concluded that Vitamin B12 may have some effective roles in pathogenesis, while folic acid deficiency cannot be considered as a prominent risk factor in OLP. ${ }^{30}$

A Study was conducted in 41 OLP Subjects to estimate folate and vitamin B12 levels by Thongprasom K, in 2001 and found low folate levels in $44 \%$ in group I and $56 \%$ in group II while serum vitamin B12 levels in both groups were within normal range with variation in folate levels. They suggested estimation of serum vitamin $B 12$ \& folate in patients with oral lesions and symptoms in OLP. ${ }^{31}$

Challacombe SJ et al in 1986 evaluated serum levels in 103 OLP patients and demonstrated anemia, low levels of iron, folate, and vitamin B12 in nine (8.7\%) $, 13(12.6 \%), 3(2.9 \%)$, and $2(1.9 \%)$ OLP patients, respectively. They suggested that all patients presenting with non-ulcerative symptoms should be screened for haematological deficiency. ${ }^{32}$

Vitamin status was assessed in 41 OLP subjects in a study conducted by Jolly $\mathrm{M}$ et al and they found improvement in 20 of 41 subjects when managed with multiple vitamin supplements. They inferred that although deficiency was significantly higher in OLP patients, it may not be the main etiology causing the OLP. ${ }^{33}$

\section{Haematological investigations ${ }^{34}$}

1. Blood picture shows Macrocytic anemia,

2. Bone marrow smear shows features of megaloblastic red blood cells,

3. Serum vitamin $B 12<160 \mathrm{ng} / \mathrm{L}$,

4. Serum folate level \& Vitamin B12 absorption testsSchilling test should be evaluated

\section{Management ${ }^{4}$}

1.Cyanocobalamin injections are administered in doses of $100-1000 \mu \mathrm{g} \mathrm{IM}$.

2. Hydroxocobalamin $1000 \mu \mathrm{g}$ given intramuscularly till a total of 5-6 mg over the duration of 3 weeks, $1000 \mu \mathrm{g}$ may be given every 3 months. ${ }^{9}$

3. Folic acid administration has been reported to reverse haematological abnormalities observed in B12 deficiency.

\section{CONCLUSION}

Nutrition plays a pivotal role maintaining the overall health status of an individual. Various vitamins and micronutrients have an impact on the integrity of oral mucosa. Thorough hematinic investigation is recommended in the management of oral lesions, particularly in patients in whom these deficiencies are prevalent. It is mandatory to identify the exact etiology of the disease and manage them accordingly .Oral lesions are among the most common early manifestations encountered by the oral physician, therefore a thorough knowledge is essential to contribute to the diagnosis and treatment of these deficiencies.

\section{REFERENCES}

1. Rajendran et al. Shafer's Textbook of Oral Pathology, Chapter 15, $5^{\text {th }}$ edition, Elsevier 2006, pp 846-6o.

2. Sharma A. Clinical; Dietetics and Nutrition. Medical Journal, Armed Forces India. 1998;54(2):176.

3. Narsinga Rao BS. Nutrient requirement and safe dietary intake for Indians. NFI Bull (Bulletin of the Nutrition Foundation of India) 2010;31:1-5

4. Rusher DR, Pawlak R. A Review of 89 Published Case Studies of Vitamin B12 Deficiency. J Hum Nutr Food Sci 2013; 1(2): 1008-48

5. Kim J, Kim MJ, Kho HS. Oral manifestations in vitamin B12 deficiency patients with or without history of gastrectomy. BMC Oral Health. 2016;16(1):60.

6. Stoopler ET, Kuperstein AS. Glossitis secondary to vitamin B12 deficiency anemia. CMAJ. 2013;185(12):E582.

7. Pontes HA, Neto NC, Ferreira KB, Fonseca FP, Vallinoto GM, Pontes FS, Pinto Ddos S., Jr. Oral manifestations of vitamin B12 deficiency: a case report. J Can Dent Assoc. 2009;75:533-7.

8. Graells J, Ojeda RM, Muniesa C, Gonzalez J, Saavedra J. Glossitis with linear lesions: an early sign of vitamin B12 deficiency. J Am AcadDermatol. 2009;60:498-500.

9. Liu HL, Chiu S, Chen KH. Effectiveness of Vitamin B12 on Recurrent Aphthous Stomatitis in Long term Care: A Systematic Review. JBLooo426 2013:11(2) 281307

10. Qazi JA .Vitamin b12 for the treatment of recurrent aphthous stomatitis. JKCD 2011;1(2):87-90 11.Kozlak S T, Walsh S J, Lalla R V. Reduced dietary intake of vitamin B12 and folate in patients with 
recurrent aphthous stomatitis. J Oral Pathol Med.2010; 39(5):420-3.

12. Volkov I, Rudoy I, Freud T et al. Effectiveness of vitamin B12 in treating recurrent aphthous stomatitis: a randomised, double-blind, placebo-controlled trial. J Am Board Fam Med 2009; 22: 9-16.

13. Carrozzo M. Vitamin B12 for the treatment of recurrent aphthous stomatitis. Evid Based Dent. 2009;10(4):114-5.

14. Burgan SZ, Sawair FA. Hematologic status in patients with recurrent aphthous stomatitis in Jordan. Amarin ZO. Saudi Med J 2006; 27 (3): 381-4.

15. Benner SE, Winn RJ, Lippman SM, Poland J, Hansen KS, et al. Regression of oral leukoplakia with alpha-tocopherol: a community clinical oncology program chemoprevention study. J Natl Cancer Ins.1993,85:44-48.

16 Stich HF, Mathew B, Sankaranarayanan R, Nair MK et al. Remission of precancerous lesions in the oral cavity of tobacco chewers and maintenance of the protective effect ofbeta- carotene or vitamin A. Am J Clin Nutr.1991, 53: 2983-3048.

17. Sankaranarayanan R, Duffy SW, Day NE, Nair MK, Padmakumary G. A casecontrolinvestigation of cancer of the oral tongue and the floor of the mouth in southernIndia. IntJ Cancer.1989,44:617-21.

18. Ramaswamy G, Rao VR, Kumaraswamy SV, AnanthaN . Serum vitamins' status in oral leucoplakias--a preliminary study. Eur J Cancer B Oral Oncol.1996,32B:120-2.

19. Richie JP Jr, Kleinman W, Marina P, Abraham P, Wynder EL, et al. Bloodiron, glutathione, and micronutrient levels and the risk of oral cancer. Nutr Cancer .2008,60:474-82.

20. Theaker JM, Porter SR, Fleming KA .Oral epithelial dysplasia in vitamin B12 deficiency. Oral Surg Oral Med Oral Pathol.1989;67:81-3.

21. Butterworth CE . Folate deficiency and cancer. In: A. Bendich, C. E. Butterworth.Editors: Micronutrients in health and disease. New York: Marcel Decker. $1991 ; 165-83$

22. oEto I, KrumdieckCL.Role of vitamin $\mathrm{B} 12$ and folate deficiencies incarcinogenesis. Adv Exp Med Biol 1986;206:313-30.

23. Khaled MA, Krumdieck CL et al. Association of folate molecules as determined byproton NMR: implications on enzyme binding. BiochemBiophys Res Commun 1985;130:1273-280.

24. Piyathilake CJ, Macaluso M, Hine RJ, Vinter DW, Richards EW, et al. (1995) Cigarettesmoking, intracellular vitamin deficiency, and occurrence of micronuclei in epithelialcells of the buccal mucosa. Cancer Epidemiol Biomarkers Prev 4:751-8.

25. Gorgulu O, Selcuk T, Ozdemir S, Sayar C, Beyazit $\mathrm{Y}$, Akbas Y. Evaluation of the roles of serum vitamin $\mathrm{B}(12)$, folate and homocysteine levels in laryngeal squamous cell carcinoma. J Int Med Res. 2010; 38 : 2047-52.

26. Almadori G, Bussu F, Galli J, Cadoni G, Zappacosta B, Persichilli S, et al. Serum levels of folate, homocysteine, and vitamin B12 in head and neck squamous cellcarcinoma and in laryngeal leukoplakia. Cancer 2005; 103:284-92.

27. Raval GN, Sainger RN, Rawal RM, et al. Vitamin $\mathrm{B}(12)$ and folate status in head and neck cancer, Asian Pac J Cancer Prev. 2002;3:155-162.

28. WangYP, Lin HP, Chen H M, Kuo YS ,Lang M J, Sun A. High frequencies of vitamin B12 and folic acid deficiencies and gastric parietal cell antibody positivity in oral submucous fibrosis patients. J Formos Med Assoc. 2015;114(9):813-9.

29. Chen HM, Wang YP, Chang JYF, Wu YC, Cheng SJ, Sun A. Significant association of deficiency of hemoglobin, iron and vitamin B12 and high homocysteine level with oral lichen planus. J Formos Med Assoc. 2015;114(2):124-9.

30. Sahebjamee M, Beitollahi JM, Mansourian A, Shahsavari N, Shabestari SB. Assessment of Serum Vitamin $\mathrm{B}_{12}$ and Folic Acid in Patients with Oral Lichen Planus: A Case Control Study. Journal of Dentistry, Shiraz University of Medical Sciences. 2010;10:36-9.

31. Thongprasom K, Youngnak P, Aneksuk V et al. Folate and vitamin B12 levels in patients with oral lichen planus, stomatitis or glossitis. Southeast Asian J Trop Med Public Health. 20o1;(32):643-7.

32. Challacombe SJ. Haematological abnormalities in oral lichen planus, candidiasis, leukoplakia and nonspecific stomatitis. Int J Oral Maxillofac Surg. 1986; 15(1):72-80.

33. Jolly M., Nobile S. Vitamin status of patients with oral lichen planus. Aust Dent J 1977; 22: 446-50.

34. Briani C, Dalla Torre C, Citton V, et al. Cobalamin deficiency: clinical picture and radiological findings. Nutrients. 2013;5(11):4521-39. 
Source of support: Nil, Conflict of interest: None declared

\section{Cite this article as:}

Alekhya K, Tejaswi K, Muppirala S, Divya D, Krishnappa SL, Arutla R. Oral Manifestations of Vitamin B12 (Cobalamin) Deficiency: A Review. Int Healthc Res J. 2019;3(1):9-15. doi: 10.26440/IHRJ/0301.04.521066

\section{AUTHOR AFFILIATIONS:}

1. Assistant Professor, Department of Oral Medicine and Radiology, MNR Dental College \& Hospital, Sangareddy-502001, Telangana, India

2. Consultant Oral \& Maxillofacial Radiologist, Department of Oral Medicine and Radiology, Max 3 G Imaging, Hyderabad-500o82

3. Consultant Oral \& Maxillofacial Radiologist, Department of Oral Medicine and Radiology, Vijaya Diagnostic Centre, Hyderabad-500034

4. Public Health Dentist, Identity Dental Hospital \& Research Center, Kadapa - 516001

5. Consultant Oral \& Maxillofacial Radiologist, Ramanagaram -562159

6. Assistant Professor, Department of Oral Medicine and Radiology,SVS Institute of Dental Sciences, Mahabubnagar, Telengana -509002

\section{*Corresponding Author:}

Dr. Kanaparthi Alekhya

Department of Oral Medicine and Radiology

MNR Dental College \& Hospital

Sangareddy-502001

Telangana India
For article enquiry/author contact details, e-mail at: editor.ihri@gmail.com 